\title{
Impedance-matched absorbers and optical pseudo black holes
}

\author{
H. Odabasi, ${ }^{1, *}$ F. L. Teixeira, ${ }^{1}$ and W. C. Chew ${ }^{2,3}$ \\ ${ }^{1}$ ElectroScience Laboratory and Department of Electrical and Computer Engineering, \\ The Ohio State University, Columbus, Ohio 43210, USA \\ ${ }^{2}$ Department of Electrical and Computer Engineering, University of Illinois at Urbana-Champaign, \\ Urbana, Illinois 61801, USA \\ ${ }^{3}$ Department of Electrical and Electronic Engineering, The University of Hong Kong, \\ Pokfulam Road, Hong Kong, China \\ *Corresponding author: odabasi.1@osu.edu
}

Received February 28, 2011; accepted March 18, 2011;

posted March 23, 2011 (Doc. ID 143195); published April 28, 2011

\begin{abstract}
We investigate the design of metamaterial blueprints for impedance-matched (reflectionless) absorbers over nonplanar surfaces in connection with optical pseudo black holes. The presence of some fundamental constraints on the existence of reflectionless absorber media at nonplanar surfaces are discussed based on analytical properties of the associated constitutive tensors on the complex $\omega$ (angular frequency) plane. In view of such constraints, some new metamaterial blueprints of near-reflectionless absorbers are suggested for integration into optical pseudo black hole devices. (C) 2011 Optical Society of America

OCIS codes: $\quad 160.1190,160.3918,260.2110,260.2710$.
\end{abstract}

\section{INTRODUCTION}

Optical (pseudo) "black hole" devices have been proposed [1-ㄷ] and built [5] based on transformation optics concepts. Most of the proposed optical black holes are devices (metamaterial shells) intended to slow down and guide incoming waves toward an inner core (while producing minimal reflections) where, depending on the intended application, electromagnetic energy can be harvested and/or efficiently absorbed (ideally, under minimal reflections as well). More generally, the term "optical black hole" can be used to denote a metamaterial device from which no impingent electromagnetic wave "returns" (or reflects from, at least in an approximate sense and for a given range of frequencies). Because no gravitational effects are implied, the term black hole is used here in a very loose sense (perhaps a more precise term is omnidirectional electromagnetic absorber [5]]). This terminology is adopted to conform with prior references on this topic [1-4]

Media derived from transformation optics typically have inhomogeneous and anisotropic permittivity $[\epsilon]$ and permeability $[\mu]$ tensors [ $[\underline{6}-\underline{10}]$. In contrast, most previously proposed media for optical black holes consisted of nonmagnetic isotropic media [2-5]. A nonmagnetic isotropic response is of interest because of the ease of fabrication, especially at optical frequencies; however, this implies that such materials are not truly impedance matched (reflectionless) and hence a low permittivity gradient (or, equivalently, an electrically large shell thickness spanning many vacuum wavelengths) is necessary to minimize reflections.

For optical black hole applications in which the main objective is not to guide incident waves to an inner core but rather to simply produce very low levels of backscattering for any incident wave direction, it is obvious that the performance crucially depends on the amount of reflection produced. With this objective in mind, we investigate here metamaterial blueprints of (near)-reflectionless absorbers for integration into black hole devices where the electrical thickness of the shell can be drastically reduced. We discuss how some of these blueprints are related to those of "perfectly matched layers" (PMLs) previously developed for numerical simulation purposes [11-14]. We point out some fundamental limitations on the existence of truly reflectionless absorbers on curved surfaces and suggest some alternatives for integrating near-reflectionless absorptive effects into metamaterial blueprints of optical black holes devices.

\section{METAMATERIAL BLUEPRINTS FOR REFLECTIONLESS ABSORBERS}

\section{A. Planar Geometries}

We derive metamaterial blueprints of reflectionless, or perfectly impedance-matched, absorbers by extending the conventional (real-valued) coordinate-transformation approach of transformation optics to complex-valued coordinate transformations. Consider, for example, the Cartesian coordinate $x$ : by transforming it via $x \rightarrow \tilde{x}=x+i \Delta_{x} / \omega$, where $\omega$ is the angular frequency and $\Delta_{x}>0$ is a continuous function of $x$, it follows that any propagating eigenfunction of the form $\exp \left(i k_{x} x\right)$ is (continuously) mapped to an exponentially decaying function of the form $\exp \left(i k_{x} \tilde{x}\right)=\exp \left(i k_{x} x-\right.$ $\left.k_{x} \Delta_{x} / \omega\right)$. This is effected without any perturbation of the boundary conditions (and, in particular, phase matching) on the normal and tangential components of the electromagnetic fields along the transverse $y-z$ plane, thus without producing reflections.

It turns out that an instance of such reflectionless absorber already exists and is provided by the so-called "anisotropicmedium" (or "uniaxial") formulation $[\underline{6}, \underline{12}, \underline{13}]$ of the PML [11], originally developed as an absorbing boundary condition for numerical simulations. In Cartesian coordinates, the PML 
material tensors that produce reflectionless absorption along $x$ are written as $[\epsilon]=\epsilon_{0}[\Lambda]_{x}$ and $[\mu]=\mu_{0}[\Lambda]_{x}$, where $[\underline{6}]$

$$
[\Lambda]_{x}=\operatorname{diag}\left\{1 / s_{x}, s_{x}, s_{x}\right\}
$$

is a diagonal tensor in Cartesian coordinates $(x, y, z)$ and $s_{x}$ is the complex-valued "stretching parameter" along the (normal) $x$ direction. Typically, $s_{x}(x)=a_{x}(x)+i \sigma_{x}(x) / \omega$, where the real part $a_{x}(x) \geq 1$ controls the phase velocity (or, equivalently, the "slowness") of the wave inside the material and the imaginary part $\sigma_{x}(x) \geq 0$ controls the amount of absorption, with the choice $\sigma_{x}(x)=0$ producing no absorption. Other more involved functional dependencies of $s_{x}$ in terms of $\omega$ are also possible [15], but we do not consider those here. Assuming the absorber is located in the half-space $x>0$, this is equivalent-from a transformation optics viewpoint- to a complexification of the spatial metric via [6]

$$
d s^{2}=d x^{2}+d y^{2}+d z^{2} \rightarrow d \tilde{s}^{2}=s_{x}^{2} d x^{2}+d y^{2}+d z^{2},
$$

which is also equivalent to an analytical continuation of the $x$ coordinate, $x \rightarrow \tilde{x}$ based on $d \tilde{x}=s_{x} d x$ or, equivalently,

$$
\tilde{x}(x)=\int_{0}^{x} s_{x}(x) \mathrm{d} x
$$

\section{B. Curved Geometries}

Equation (1) corresponds to the desired material blueprint for a planar reflectionless absorber. For applications in optical black hole devices, it is desirable to extend the reflectionless property to curved surfaces. In cylindrical coordinates $(\rho, \phi, z)$, for example, a similar complexification on the spatial metric along the differential element in the radial direction $\rho$ would produce

$$
d s^{2}=d \rho^{2}+\rho^{2} d \phi^{2}+d z^{2} \rightarrow d \tilde{s}^{2}=s_{\rho}^{2} d \rho^{2}+\rho^{2} d \phi^{2}+d z^{2},
$$

where now $s_{\rho}=a_{\rho}(\rho)+i \sigma_{\rho}(\rho) / \omega$ represents the complex stretching parameter along $\rho$. Note that the (real-valued) choice

$$
s_{\rho}^{2}=\left(1-\frac{L}{\rho}\right)^{-1}
$$

i.e., $a_{\rho}(\rho)=(1-L / \rho)^{-1 / 2}$ and $\sigma_{\rho}(\rho)=0$, with $L>0$, recovers the parameters of an optical Schwarzschild black hole device as considered in [3].

Using transformation optics techniques, it is easy to show [6] that the above is equivalent to a change on the constitutive tensors of the background medium to $[\epsilon]=\epsilon_{0}[\Lambda]_{\rho}$ and $[\mu]=\mu_{0}[\Lambda]_{\rho}$, where

$$
[\Lambda]_{\rho}=\operatorname{diag}\left\{1 / s_{\rho}, s_{\rho}, s_{\rho}\right\}
$$

in a cylindrical coordinate basis $(\rho, \phi, z)$ representation. However, unlike the planar case [Eq, (1)], the resulting medium ceases to be reflectionless, with a reflection coefficient that now depends on the radius of curvature [16]. A truly reflectionless media in a cylindrical surface entails applying a transformation on the radial coordinate $\rho$ (and not simply on the radial metric element) [14]. In other words, assuming an interface at $\rho=\rho_{0}$, let

$$
\tilde{\rho}(\rho)=\rho_{0}+\int_{\rho_{0}}^{\rho} s_{\rho}(\rho) \mathrm{d} \rho=b_{\rho}(\rho)+i \Delta_{\rho}(\rho) / \omega
$$

for a point $\rho$ in the interior of the absorber medium, with

$$
\begin{gathered}
b_{\rho}(\rho)=\rho_{0}+\int_{\rho_{0}}^{\rho} a_{\rho}(\rho) \mathrm{d} \rho, \\
\Delta_{\rho}(\rho)=\int_{\rho_{0}}^{\rho} \sigma_{\rho}(\rho) \mathrm{d} \rho .
\end{gathered}
$$

Note that because both $a_{\rho}(\rho)$ and $\sigma_{\rho}(\rho)$ are bounded, $b_{\rho}(\rho)$ and $\Delta_{\rho}(\rho)$ are continuous functions of the variable $\rho$ by construction. The corresponding material tensors, derived using transformation optics techniques [14], read as

$$
[\Lambda]_{(\rho, \phi)}=\operatorname{diag}\left\{\tilde{\rho} /\left(s_{\rho} \rho\right),\left(s_{\rho} \rho\right) / \tilde{\rho},\left(s_{\rho} \tilde{\rho}\right) / \rho\right\}
$$

in a cylindrical coordinate basis. Metamaterial blueprints of reflectionless absorbers for spherical or more general (doubly curved) surfaces also exist [17]. Note that these blueprints are three-dimensional and equally valid for any polarization.

\section{MATERIAL TENSOR PROPERTIES ON CONVEX AND CONCAVE SURFACES}

One important distinction between the reflectionless absorber tensors discussed above for planar surfaces [Eq. (1)] and for curvilinear surfaces [Eq. (10)] is the presence of the radial parameters $\rho$ and $\tilde{\rho}$ in the latter. These radial parameters lead to a fundamental difference between the corresponding material properties on convex and concave surfaces. Before examining this difference, we should first recall that constitutive tensors of causal passive media are free from singularities on the upper half of the complex $\omega$ plane [18]; in particular, they can only exhibit poles on the real axis or the lower half of the complex $\omega$ plane. This property is a condition, for example, for the derivation of Kramers-Kronig relations, which relate the real and imaginary part of a (causal, passive) constitutive tensor [18].

Let us briefly examine the singularities of the constitutive tensors $[\Lambda]_{x}$ and $[\Lambda]_{(\rho, \phi)}$ on the complex $\omega$ plane. For $[\Lambda]_{x}$, it is clear that the only singularity is due to the pole at $\omega_{x}=-i \sigma_{x}(x) / a_{x}(x)$. Since $a_{x}(x)$ and $\sigma_{x}(x)$ are both nonnegative, this pole is located either on the lower half-plane or on the real axis. On the other hand, $[\Lambda]_{(\rho, \phi)}$ has two poles: one at $\omega_{\rho}^{(1)}=-i \sigma_{\rho}(\rho) / a_{\rho}(\rho)$ and the other at $\omega_{\rho}^{(2)}=-i \Delta_{\rho}(\rho) / b_{\rho}(\rho)$. Again, because $a_{\rho}(\rho)$ and $\sigma_{\rho}(\rho)$ are nonnegative, the pole $\omega_{\rho}^{(1)}$ is located either on the lower half-plane or on the real axis. However, the location of the pole $\omega_{\rho}^{(2)}$ depends on the sign of $\Delta_{\rho}(\rho)$. Taking the integral in Eq. (9) for $\rho>\rho_{0}$ (i.e., outwardly in the radial direction, which means that the cylindrical interface of the PML medium is concave, as seen from the "outside" medium) leads to $\Delta_{\rho}(\rho)>0$. In contrast, taking the integral in Eq. (9) for $\rho<\rho_{0}$ (i.e., inwardly in the radial direction, which means that the cylindrical surface is convex, as seen from the "outside") leads to $\Delta_{\rho}(\rho)<0$. The condition $\rho>$ $\rho_{0}$ is the typical geometry present in numerical simulations where the reflectionless PML medium is inserted at the outer boundary of the domain. Conversely, the condition $\rho<\rho_{0}$ is the case of interest for scenarios in which such media are to 


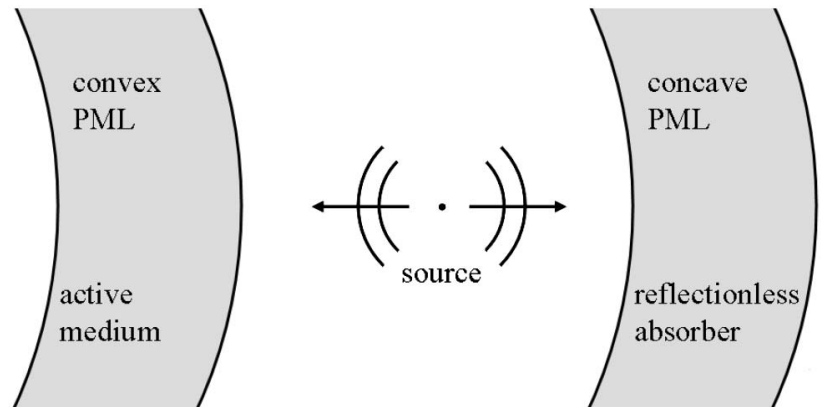

Fig. 1. Behavior of PML-derived metamaterial blueprints on concave versus convex surfaces.

be used for coating of finite objects, such as for optical black hole devices.

If $\Delta_{\rho}(\rho) \geq 0$, the tensor $[\Lambda]_{(\rho, \phi)}$ is singularity free on the upper half-plane. On the other hand, if $\Delta_{\rho}(\rho)<0$, the pole $\omega_{\rho}^{(2)}$ is present on the upper half-plane. This presence of a pole in the upper half-plane implies that the medium is not an absorber anymore: by examining the impulse response $[\Lambda]_{(\rho, \phi)}(t)$ given as

$$
[\Lambda]_{(\rho, \phi)}(t)=\mathcal{F}^{-1}\left[[\Lambda]_{(\rho, \phi)}(\omega)\right]
$$

for $\Delta_{\rho}(\rho) \leq 0$ and where $\mathcal{F}$ denotes a Fourier transform, it is easy to verify $[\underline{19}, \underline{20}]$ that $[\Lambda]_{(\rho, \phi)}(t)$ exhibits exponential growth in time due to $\omega_{\rho}^{(2)}$. This behavior is illustrated in Section 4 and characterizes an active media (as opposed to an absorber media, which would provide exponential decay on $\left.[\Lambda]_{(\rho, \phi)}(t)\right)$. These two situations are summarized in Fig. 1 .

\section{METAMATERIAL BLUEPRINTS OF NEAR- REFLECTIONLESS ABSORBERS ON CONVEX SURFACES}

Because of the above limitation, we discuss next some alternatives for obtaining near-reflectionless anisotropic absorbers on convex surfaces (left side of Fig. 1).

\section{A. Approximate "PML-Derived" Metamaterial Blueprints} A possible approach to obtain metamaterial blueprints of near-reflectionless absorbers on convex surfaces is to employ approximate PML models. For example, the tensor model in Eq. (10) can still be formally employed on convex surfaces as long as, instead of using Eq. (9), a different choice for $\Delta_{\rho}(\rho)$ is made where the condition $\Delta_{\rho}(\rho) \geq 0$ is enforced in an $a d$ hoc fashion (e.g., by "inverting" the sign in front of the integral in Eq. (9) or "setting" that integral to zero). The choice $\Delta_{\rho}(\rho)=0$, for example, would correspond to Eq. (4). Such material blueprints are near-reflectionless in the sense that the magnitude of the reflection coefficient is not exactly zero but can be made arbitrarily small by increasing the (electrical size of the) radius of curvature-in the limit of electrically large radius, the reflection goes to zero as Eq. (10) recovers Eq. (1).

\section{B. Simulations and Backscattering Results}

Figure 2 illustrates the effect of such metamaterial absorbers in suppressing reflections. It shows, for two time instants, the "snapshot" in the $x-y$ plane of the electric field produced by a $z$-directed point source (electric dipole) in the presence of a nearby perfect electric conductor (PEC) circular cylinder.
The PEC cylinder is coated with a metamaterial "shell" with $[\epsilon]=\epsilon_{0}[\Lambda]_{(\rho, \phi)}$ and $[\mu]=\mu_{0}[\Lambda]_{(\rho, \phi)}$, with $[\Lambda]_{(\rho, \phi)}$ given by Eq. (10) and where $\Delta_{\rho}$ is chosen in one of two ad hoc ways: (1) as given by minus the integral in Eq. (9) $\left(\Delta_{\rho}>0\right)$ or (2) set to zero, $\Delta_{\rho}=0$. Also shown is the field distribution with no absorber present. The point source is located $12.5 \mathrm{~cm}$ away from the surface of the coating and is excited by a short pulse with center frequency $300 \mathrm{MHz}$. The PEC cylinder has a $1 \mathrm{~m}$ radius and the coating has a $0.5 \mathrm{~m}$ thickness. A PEC cylinder is used as the "core," as this provides the worst-case scenario for evaluating the efficacy of the absorber shell in reducing reflection. Note that the shell thickness is half of the (vacuum) wavelength, whereas the thicknesses of previously proposed (nonabsorptive) black hole shells span many wavelengths. This result is obtained using a three-dimensional finitedifference time-domain (FDTD) algorithm. To eliminate staircasing error in the circular geometry, we employ a cylindrical grid [21] instead of a conventional (Cartesian) FDTD grid.

Figure 2 serves to illustrate, at a qualitative level, the effectiveness of the absorber shell on suppressing the reflected field, with both choices $\Delta_{\rho}>0$ and $\Delta_{\rho}=0$ yielding similar performance. A more detailed (quantitative) analysis of the reflection coefficient is presented below. On the other hand, Fig. 3 shows the scattering from the same geometry except that the metamaterial shell has $[\Lambda]_{(\rho, \phi)}$ with negative $\Delta_{\rho}$ as computed from Eq. (9). Note that, although the frontal reflection is still strongly suppressed at first, Fig. 3 clearly shows the onset of the spurious field growth inside the shell due to the upper half-plane pole, as discussed in Section $\underline{3}$.

For realizations of such absorbers based on annular strips (columns) of periodic inclusions, $\sigma_{\rho}$ should assume a stepwise distribution behavior along $\rho$ [5]. The amount of reflection in this case depends on the conductivity contrast between adjacent strips and increases for larger contrasts (an analogous issue exists for $a_{\rho}$ as well as for PML models on discrete grids [22]). To minimize reflections, it is therefore desirable to adopt a tapered profile for $\sigma_{\rho}(\rho)$, such as $\sigma_{\rho}(\rho)=\left(\sigma_{0} / \epsilon_{0}\right) \times$ $\left|\left(\rho-\rho_{0}\right) / \delta\right|^{p}$, where $\sigma_{0}$ is the maximum value of the conductivity (in siemens per meter), $\epsilon$ is the vacuum permittivity (in farads per meter), $\delta$ is the overall absorber thickness, and $p$ represents the degree of the taper $[\underline{16}, \underline{19}]$.

To quantify the level of residual reflections produced, we plot the "reflection" or "backscattering coefficient" as a function of time in Fig. 4. This coefficient is computed here in the following way: the $z$ component of the electric field is first sampled at a point $3.125 \mathrm{~cm}$ away from the coating surface (along the line segment from the origin to the source location) with and without the presence of the coated cylinder; the reflection coefficient is then computed as the (magnitude of the) difference between these two field values normalized by the peak field value observed at this same sample point. Any contribution to this coefficient originates from the residual backscattering. The results shown in Fig. $\underline{4}$ assume 32 annular strips for the coating (so that each strip has a thickness of about $1.5 \mathrm{~cm}$ ) and a tapered profile for $\sigma_{\rho}$ with $p=4$. For simplicity, we set $a_{\rho}=1$. Three results are shown for different values of $\sigma_{0}$. These results serve to illustrate a basic tradeoff when attempting to minimize the reflection: larger $\sigma_{0}$ values increase the early-time "front-end" reflection due to the (larger) contrast present between successive strips but decrease the late-time "back-end" reflection from the PEC 


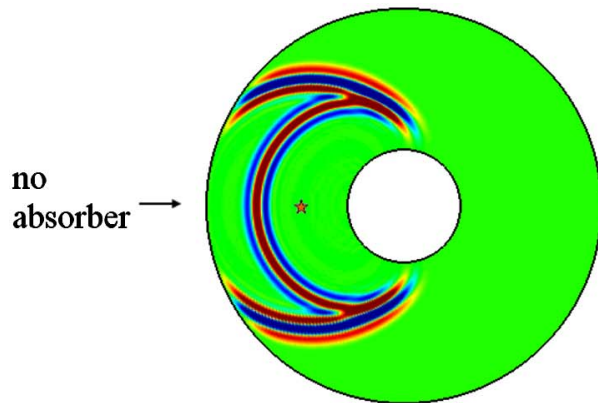

$\mathrm{t}=8.79 \mathrm{~ns}$

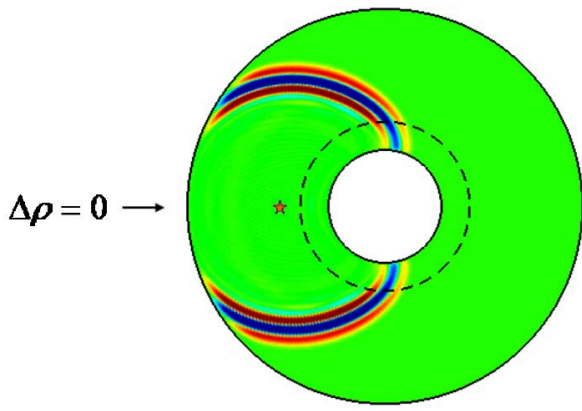

$\mathrm{t}=8.79 \mathrm{~ns}$

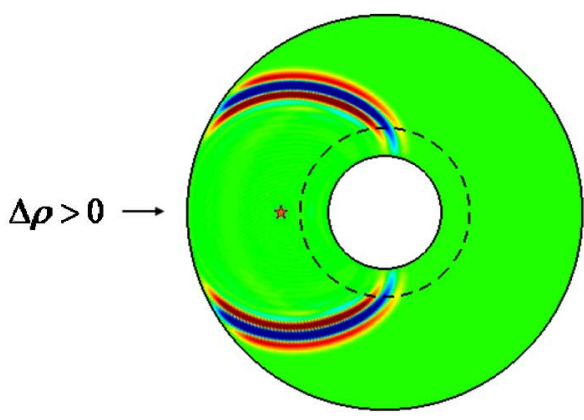

$\mathrm{t}=8.79 \mathrm{~ns}$

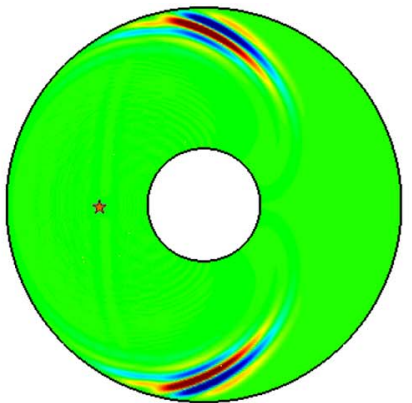

$\mathrm{t}=13.48 \mathrm{~ns}$

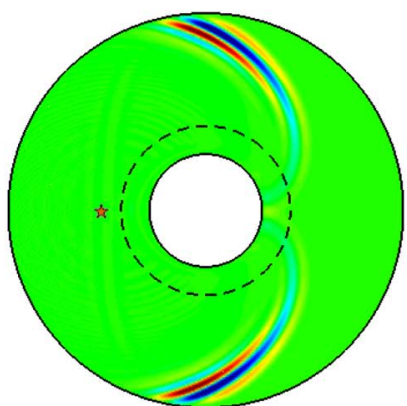

$\mathrm{t}=13.48 \mathrm{~ns}$

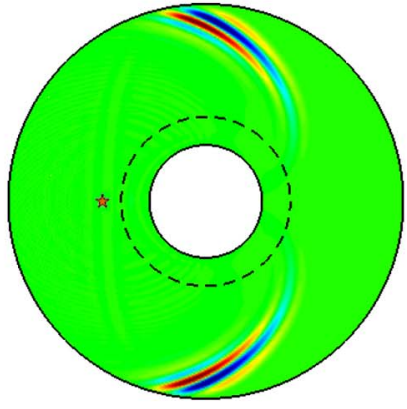

$\mathrm{t}=13.48 \mathrm{~ns}$

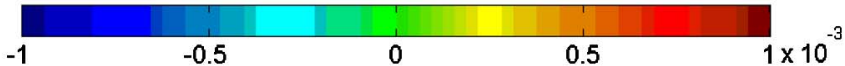

Fig. 2. (Color online) Spatial distribution, for two instants of time, of the electric field produced by a point source (with location indicated by the star symbol) nearby a circular PEC cylinder (in white). In the top sequence, the PEC cylinder is stand alone, producing a sharp reflection (backscattering). In the middle and bottom sequences, the PEC cylinder is coated by a metamaterial absorber shell (whose outer boundary is indicated by a dashed circle) having constitutive parameters $[\epsilon]=\epsilon_{0}[\Lambda]_{(\rho, \phi)}$ and $[\mu]=\mu_{0}[\Lambda]_{(\rho, \phi)}$, with $[\Lambda]_{(\rho, \phi)}$ given by Eq. (10) and where the parameter $\Delta \rho$ is enforced to be zero or positive in an ad hoc fashion. These two choices are both very effective in suppressing reflections (backscattering).

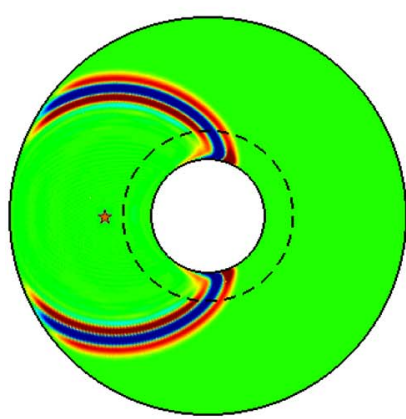

$\mathrm{t}=8.79 \mathrm{~ns}$

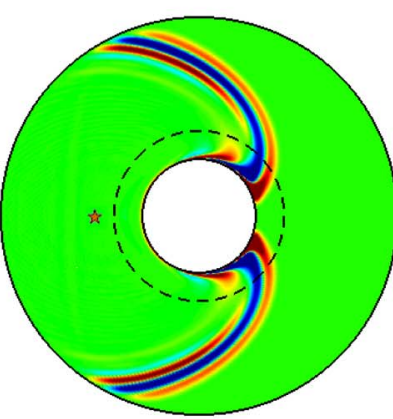

$\mathrm{t}=11.72 \mathrm{~ns}$

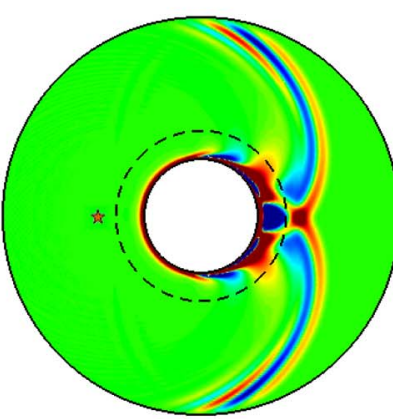

$\mathrm{t}=14.66 \mathrm{~ns}$

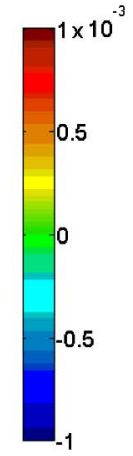

Fig. 3. (Color online) Same scattering geometry as before except that the metamaterial coating used has $[\Lambda]_{(\rho, \phi)}$ as given by Eq. (10) with negative parameter $\Delta_{\rho}$ as directly computed from Eq. (9). Three snapshots of the electric field distribution are presented. Although the frontal reflection is still strongly suppressed, the spurious field growth is clearly visible at the $t=11.72 \mathrm{~ns}$ and $t=14.66 \mathrm{~ns}$ snapshots, with a particularly strong onset in the "shadow region." 


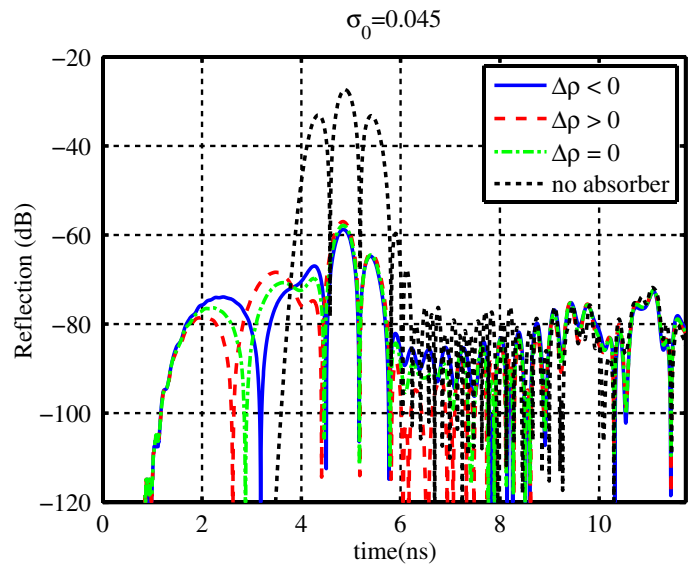

(a)

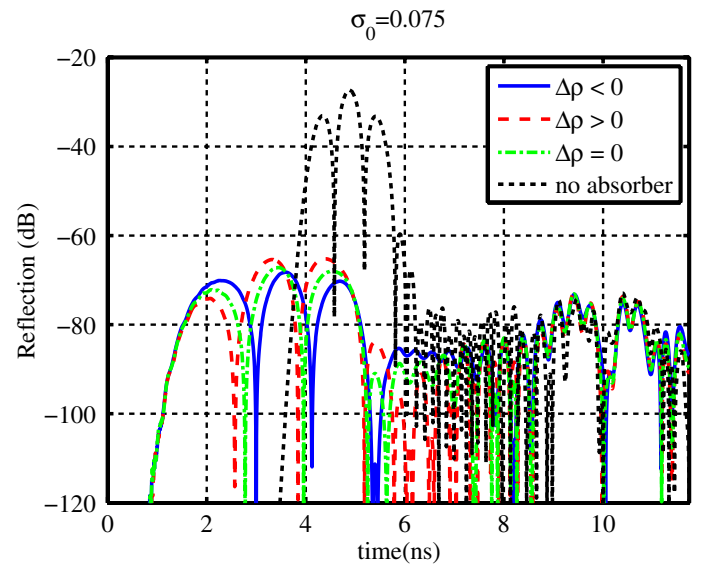

(b)

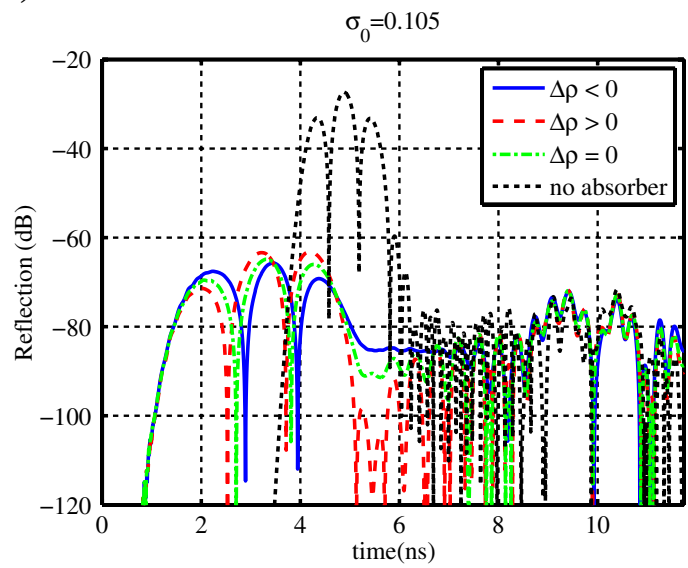

(c)

Fig. 4. (Color online) Reflection coefficients computed for different choices of $\Delta_{\rho}$, as in Fig. 2 , and three values of the conductivity $\sigma_{0}$ factor: (a) $\sigma_{0}=0.045$, (b) $\sigma_{0}=0.075$, and (c) $\sigma_{0}=0.105$. Lower $\sigma_{0}$ values produce less reflection from the front end of the coating (at about $2 \mathrm{~ns}$ ) but more reflection from the PEC cylinder itself (at about $5 \mathrm{~ns}$ ). Conversely, larger $\sigma_{0}$ values decrease the reflection originated from the PEC cylinder but produce more reflection from the front end of the coating. The reflection from the PEC cylinder with no coating ("no absorber") is also plotted for comparison.

cylinder itself (residual after a "two-way travel" within the absorber); conversely, lower $\sigma_{0}$ values decrease the conductivity contrast between successive annular strips (hence the frontend reflection) but increase the back-end reflection from the PEC cylinder itself as the field absorption becomes less pronounced during the two-way travel within the absorber.

Note that the reflection coefficient does not show any marked degradation by considering $\Delta_{\rho}>0$ or $\Delta_{\rho}=0$ as opposed to $\Delta_{\rho}<0$. Although the reflection levels presented in Fig. $\underline{4}$ are characteristic of a particular choice of conductivity profile and number of "inclusion strips," they are good indicators of the relative practical performance among the three $\Delta_{\rho}$ choices considered. The spurious field growth for $\Delta_{\rho}<0$ is present but not visible within the time window shown in Fig. 4 . Next, Fig. $\underline{5}$ shows a set of results with larger $\sigma_{0}$, where this spurious growth appears earlier and is visible within this time window.

\section{Optical Black Hole Metamaterial Blueprints with Embedded Absorption}

The near-reflectionless metamaterial model considered above can be used either as a "stand-alone" coating (as simulated) or as the absorber core inside a conventional (nonabsorbing) black hole shell. In the latter scenario, the constitutive tensors of the absorber core read as $[\epsilon]=\epsilon_{I}[\Lambda]_{(\rho, \phi)}$ and $[\mu]=\mu_{I}[\Lambda]_{(\rho, \phi)}$, with $[\Lambda]_{(\rho, \phi)}$ given by Eq. (10) under the condition $\Delta_{\rho}(\rho) \geq 0$, with $\epsilon_{I}$ and $\mu_{I}$ representing the values of the permittivity and permeability, respectively, of the inner boundary of the (nonabsorbing) shell.

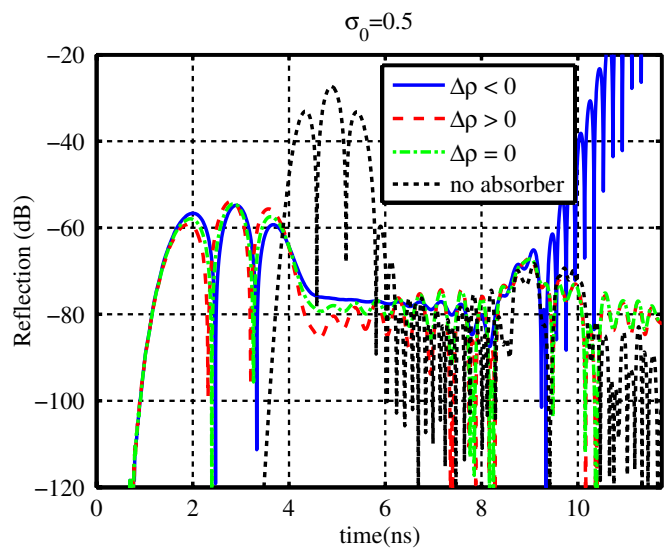

Fig. 5. (Color online) Reflection coefficient results for $\sigma_{0}=0.5$, showing the spurious field growth (at about $10 \mathrm{~ns}$ ) originating from the metamaterial coating with $\Delta_{\rho}<0$. 
Alternatively, a metamaterial blueprint can be designed to effect absorption throughout the black hole medium. To show this, let us first write the spatial component of the metric of an optical Schwarzschild (nonabsorbing) black hole device, given by [3]

$$
d s^{2}=\frac{1}{1-L / \rho} d \rho^{2}+\rho^{2} d \phi^{2}+d z^{2},
$$

which can also be written as

$$
d s^{2}=d \bar{\rho}^{2}+\rho^{2} d \phi^{2}+d z^{2}
$$

by using the change of variables $\rho \rightarrow \bar{\rho}$, where

$$
\begin{aligned}
\bar{\rho}(\rho)= & \rho_{0}+\left(\rho \sqrt{1-L / \rho}-\rho_{0} \sqrt{1-L / \rho_{0}}\right) \\
& +\frac{1}{2} L \ln \left(\frac{2 \rho(1+\sqrt{1-L / \rho})-L}{2 \rho_{0}\left(1+\sqrt{1-L / \rho_{0}}\right)-L}\right)
\end{aligned}
$$

for $\rho \leq \rho_{0}$ and $\bar{\rho}=\rho$ for $\rho>\rho_{0}$. Here, $\rho_{0}$ is the radius of the device and $L$ is a positive parameter ("Schwarzschild radius") such that $L<\rho_{0}$. Note that $\bar{\rho}$ decreases monotonically as $\rho$ decreases in the interval $L<\rho<\rho_{0}$ and can assume negative values, with $\bar{\rho} \rightarrow-\infty$ for $\rho \rightarrow L$. The thickness $t$ of the optical black hole device is chosen so that $t<\rho_{0}-L$ to avoid the singularity at $\rho=L$. In particular, an optical black hole device with thickness $t$ can be used to coat an object with radius $b=\rho_{0}-t$ with $L<b<\rho_{0}$.

The generalized metamaterial blueprint for an optical Schwarzschild black hole with an embedded nearreflectionless absorption mechanism reads

$$
d s^{2}=\left(\frac{1}{\sqrt{1-L / \rho}}+i \frac{\sigma_{\rho}(\rho)}{\omega}\right)^{2} d \rho^{2}+\rho^{2} d \phi^{2}+d z^{2},
$$

which is in the same generic form of Eq. (4) but now with

$$
s_{\rho}(\rho)=\frac{1}{\sqrt{1-L / \rho}}+i \frac{\sigma_{\rho}(\rho)}{\omega}
$$

so that corresponding material tensors are written as $[\epsilon]=$ $\epsilon[\Lambda]_{\rho}$ and $[\mu]=\mu[\Lambda]_{\rho}$ with $[\Lambda]_{\rho}$ given by Eq. ( $\left.\underline{6}\right)$ in a cylindrical coordinate basis and with $s_{\rho}$ now given by Eq. (타).

\section{CONCLUSION}

We have discussed the derivation of metamaterial blueprints for (near)-reflectionless absorbers and their possible integration into optical pseudo black hole devices. Fundamental theoretical constraints on truly reflectionless absorption models at convex surfaces were pointed out and explained by studying the analyticity of the associated constitutive tensors in the complex $\omega$ plane. In view of such constraints, "tweaked" nearreflectionless absorber blueprints were proposed. Simulation results of such absorber models have shown their effectiveness in suppressing backscattering. Some basic trade-offs in the absorber performance stemming from the use of a stepwise conductivity distribution along the radial direction were also pointed out by examining the residual backscattering produced by different conductivity profiles.

This paper focused the analysis at the "blueprint" (constitutive tensor description) level. It is beyond the objectives of this paper to delve into fabrication issues. Suffice to say that the proposed absorbers exhibit a layered structure that can facilitate eventual fabrication. Integration of spatially varying conductivity can be done, at least in the microwave frequency range, by means of stacking resistive sheets (R-cards) in decreasing resistivity values from the outer to the inner shell (such as in some radar absorber designs) and using a variable density of lossy magnetic particles, for example.

\section{APPENDIX A: EXPLORING METRIC CURVATURE FOR EMBEDDED ABSORPTION}

In this Appendix, we briefly consider another possible venue for incorporating absorption into metamaterial blueprints; however, because the resulting constitutive tensors are more intricate, they are likely to pose additional challenges for fabrication.

The origin of the asymmetry in the behavior of the PML-like tensors on curved surfaces described in Section 3 can be traced from the fact that, for concave surfaces (Fig. 1, right), the metric factor $\rho$ associated with the $d \phi$ differential element-which sets the circumference length along the azimuth as $C(\rho)=2 \pi \rho$ in flat space-increases monotonically along the direction of complex radial stretching. As seen before, if the latter has a positive imaginary part (viz. $\sigma_{\rho}>0$ ) in Eq. (9) to yield absorption, the former will have it likewise (viz. $\bar{\Delta}_{\rho}>0$ ). Conversely, on convex surfaces (Fig. 1 , left), the metric factor $\rho$ decreases in the direction of complex radial stretching, leading to $\Delta_{\rho}<0$ if $\sigma_{\rho}>0$ and hence active media behavior as demonstrated in Section $\underline{4}$.

It is possible to restore absorptive behavior in the convex case by employing a metamaterial where the (local) electrical length of the circumference $C(\rho / \lambda) \neq 2 \pi \rho / \lambda$ and the function $C(\rho / \lambda)$ is "designed" not to decrease monotonically for decreasing argument $\rho$. This can be achieved, for example, by the following generalization of Eqs. (12) and (13):

$$
d s^{2}=d \bar{\rho}^{2}+\left(B^{2}+\bar{\rho}^{2}\right) d \phi^{2}+d z^{2},
$$

which implies $C(\rho)=2 \pi \sqrt{B^{2}+\bar{\rho}^{2}}$, where $B$ is a parameter that sets the "minimum" length of the circumference at $\bar{\rho}=0$. From Eq. (14), in terms of the original (physical) radial variable $\rho$, the minimum circumference occurs when $\rho$ satisfies the equation

$$
\begin{aligned}
\frac{L}{2} \ln \left[\frac{2 \rho(1+\sqrt{1-L / \rho})-L}{2 \rho_{0}\left(1+\sqrt{1-L / \rho_{0}}\right)-L}\right]= & \left(\rho_{0} \sqrt{1-L / \rho_{0}}\right. \\
& -\rho \sqrt{1-L / \rho})-\rho_{0}
\end{aligned}
$$

The parameter $B$ is chosen such that $B<b$. The effect of the metric Eq. (A1) can be illustrated by sketching its corresponding "embedding diagram" [23] (in a three-dimensional space) of a (two-dimensional) annular region of space (shell) with the two-dimensional version of Eq. (A1), i.e., $d s^{2}=d \bar{\rho}^{2}+\left(B^{2}+\right.$ $\left.\bar{\rho}^{2}\right) d \phi^{2}$, as shown in Fig. 6(a). This diagram depicts the relative "electrical" distances inside the associated metamaterial (using the local wavelength as the fundamental length scale). The top boundary of Fig. 6(a) corresponds to the outer boundary of the corresponding metamaterial shell; the bottom 


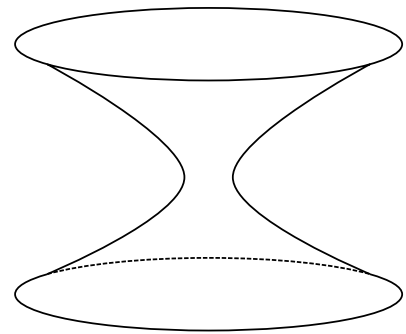

(a)

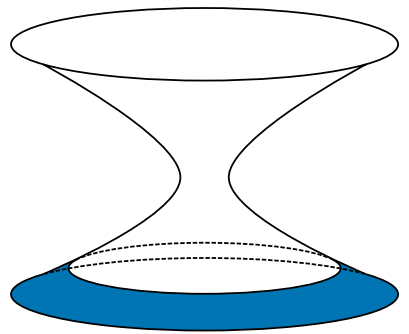

(b)
Fig. 6. (Color online) (a) Embedding diagram representing the electrical dimensions inside a metamaterial region of annular shape corresponding to the metric in Eq. (15). The top boundary represents the circumference (electric length) of the outer boundary of the metamaterial shell. The bottom boundary represents the same for the inner boundary of the metamaterial shell (although the top and bottom boundaries have the same electrical sizes, they do not need to be so in general). (b) Similar embedding diagram with the shaded area showing a possible PML-like region.

boundary represents the inner boundary of the shell and is connected to the object/region to be coated. The smallest radius in Fig. 6(a) corresponds to $\bar{\rho}=0$. The key feature that allows for $C(\overline{\rho / \lambda}) \neq 2 \pi \rho / \lambda$ is the presence of a nonzero curvature (Riemann tensor) induced by this metric. The general shape of this embedding diagram shows a superficial resemblance to (a section of) the embedding diagram of a Schwarzschild "wormhole" (or "Einstein-Rosen bridge") [23]. Note that electromagnetic wormhole devices [24] have actually been proposed before; those proposals are of a different nature and purpose than what is considered here.

Using transformation optics techniques [6] , it is easy to show that the constitutive tensors of the metamaterial equivalent to the metric in Eq. ( $\underline{\text { A1) }}$ are written as $[\epsilon]=\epsilon_{0}[\Lambda]_{C}$ and $[\mu]=\mu_{0}[\Lambda]_{C}$, with
3. H. Chen, R.-X. Miao, and M. Li, "Transformation optics that mimics the system outside a Schwarzschild black hole," Opt. Express 18, 15183-15188 (2010).

4. C. Argyropoulos, E. Kallos, and Y. Hao, "FDTD analysis of the optical black hole," J. Opt. Soc. Am. 27, 2020-2025 (2010).

5. Q. Cheng, T. J. Cui, W. X. Jiang, and B. G. Cai, "An omnidirec tional electromagnetic absorber made of metamaterials," New J. Phys. 12, 063006 (2010).

6. F. L. Teixeira and W. C. Chew, "Differential forms, metrics, and the reflectionless absorption of electromagnetic waves," J. Electromagn. Waves Appl. 13, 665-686 (1999).

7. J. B. Pendry, D. Schurig, and D. R. Smith, "Controlling electromagnetic fields," Science 312, 1780-1782 (2006).

8. O. Ozgun and M. Kuzuoglu, "Electromagnetic metamorphosis: reshaping scatterers via conformal anisotropic metamaterial coatings," Microw. Opt. Technol. Lett. 49, 2386-2392 (2007).

9. O. Ozgun and M. Kuzuoglu, "Utilization of anisotropic metamaterial layers in waveguide miniaturization and transitions," IEEE Microw. Wirel. Compon. Lett. 17, 754-756 (2007).

10. B. Donderici and F. L. Teixeira, "Metamaterial blueprint for reflectionless waveguide bends," IEEE Microw. Wirel. Compon. Lett. 18, 233-235 (2008).

11. J. P. Berenger, "A perfectly matched layer for the absorption of electromagnetic waves," J. Comput. Phys. 114, 185-200 (1994).

12. Z. S. Sacks, D. M. Kingsland, R. Lee, and J.-F. Lee, "A perfectly matched anisotropic absorber for use as an absorbing boundary condition," IEEE Trans. Antennas Propag. 43, 1460-1463 (1995).

13. R. W. Ziolkowski, "The design of Maxwellian absorbers for numerical boundary conditions and for practical applications using artificial engineered materials," IEEE Trans. Antennas Propag. 45, 656-671 (1997).

14. F. L. Teixeira and W. Chew, "Systematic derivation of anisotropic PML absorbing media in cylindrical and spherical coordinates," IEEE Microw. Guided Wave Lett. 7, 371-373 (1997).

15. M. Kuzuoglu and R. Mittra, "Frequency dependence of the constitutive parameters of causal perfectly matched anisotropic absorbers," IEEE Microw. Guided Wave Lett. 6, 447-449 (1996).

16. M. Kuzuoglu and R. Mittra, "Investigation of nonplanar perfectly matched absorbers for finite-element mesh truncation," IEEE Trans. Antennas Propag. 45, 474-486 (1997).

$$
[\Lambda]_{C}=\operatorname{diag}\left\{\left[\frac{(\rho-L)\left(B^{2}+\bar{\rho}(\rho)^{2}\right)}{\rho^{3}}\right]^{1 / 2},\left[\frac{\rho^{3}}{(\rho-L)\left(B^{2}+\bar{\rho}(\rho)^{2}\right)}\right]^{1 / 2},\left[\frac{B^{2}+\bar{\rho}(\rho)^{2}}{\rho(\rho-L)}\right]^{1 / 2}\right\}
$$

in a cylindrical coordinate basis and with $\bar{\rho}(\rho)$ as given in Eq. (14). Since $C(\rho)$ grows for $\rho<\rho_{h}$, a complex PML-like coordinate transformation on $\rho$ can be effected within the region $b<\rho<\rho_{h}$ without causing exponential growth in the fields, as illustrated by the shaded region in Fig. 6(b).

\section{ACKNOWLEDGMENTS}

We thank W. Sha from the University of Hong Kong for useful comments.

\section{REFERENCES}

1. U. Leonhardt and T. G. Philbin, "General relativity in electrical engineering," New J. Phys. 8, 247 (2006).

2. E. E. Narimanov and A. V. Kildishev, "Optical black hole: broadband omnidirectional light absorber," Appl. Phys. Lett. 95, 041106 (2009).
17. F. L. Teixeira and W. C. Chew, "Analytical derivation of a conformal perfectly matched absorber for electromagnetic waves," Microw. Opt. Technol. Lett. 17, 231-236 (1998).

18. W. C. Chew, Waves and Fields in Inhomogeneous Media (Van Nostrand, 1995).

19. F. L. Teixeira and W. C. Chew, "On causality and dynamic stability of perfectly matched layers for FDTD simulations," IEEE Trans. Microw. Theory Tech. 47, 775-785 (1999).

20. F. L. Teixeira, "On aspects of the physical realizability of perfectly matched absorbers for electromagnetic waves," Radio Sci. 38, 8014 (2003).

21. F. L. Teixeira and W. C. Chew, "Finite difference computation of transient electromagnetic waves for cylindrical geometries in complex media," IEEE Trans. Geosci. Remote Sens. 38, 1530-1543 (2000).

22. W. C. Chew and J. M. Jin, "Perfectly matched layers in the discretized space: an analysis and optimization," Electromagnetics 16, 325-340 (1996).

23. C. W. Misner, K. S. Thorne, and J. A. Wheeler Gravitation (Freeman and Company, 1973).

24. A. Greenleaf, Y. Kurylev, M. Lassas, and G. Uhlmann, "Electromagnetic wormholes and virtual magnetic monopoles from metamaterials," Phys. Rev. Lett. 99, 183901 (2007). 